\title{
Snapshot of Energy Optimization Techniques to Leverage Life of Wireless Sensor Network
}

\author{
Kavya A P ${ }^{1}$ \\ Assistant Professor \\ Dept. of Electronics and Communication Engineering \\ Vidyavardhaka College of Engineering \\ Mysuru, Karnataka, India
}

\author{
Dr. D J Ravi ${ }^{2}$ \\ Professor \\ Dept. of Electronics and Communication Engineering \\ Vidyavardhaka College of Engineering \\ Mysuru, Karnataka, India
}

\begin{abstract}
Energy Optimization in Wireless Sensor Network (WSN) deals with the techniques which targets higher degree of energy efficiency using resource-constraint sensor nodes with minimal inclusion of any different variants of resources. At present, there are various approaches and techniques towards addressing the problems of energy but not all the research implications can be considered as optimized approach. Therefore, this paper reviews all the existing energy optimization schemes, categorizes them, briefly discuss about their strength and weakness to offer a compact snapshot of existing energy optimization techniques in WSN. The paper also contributes towards exploring the updates research trends and highlights about the open-end research problems in WSN. It is anticipated that the study findings of this manuscript will offer a true picture of study effectiveness in dealing with energy challenges so that favorable direction of investigation towards evolving up optimized solution comes up with promising outcome.
\end{abstract}

Keywords-Battery; energy efficiency; energy optimization; network lifetime; sensor node; wireless sensor network

\section{INTRODUCTION}

Wireless Sensor Network (WSN) has been used for long time towards remote sensing various attributes of physical environment [1]. One of the prime beneficial factors of relying on WSN is its capability to work in harsh environment which are inaccessible to humans. There are various applications in WSN and majority of them demands to have an extensive life time [2]-[5]. In this regard, optimizing the energy attribute has become one of the significant concerns among the researchers as well as users in the field of WSN. The primary reason for interest in energy optimization is because of limited lifetime of external batteries within this sensing device [6]. Usually, this external battery offers the direct power for all the internal and external operations to be carried out by a sensing device in WSN. After the battery is exhausted, they are usually discarded and the sensors are considered to be operationally dead. However, a recent concept of energy harvesting allows the node to harvest energy and achieve power from different sources which can recharge the battery [7][8]. Although this idea is impressive, but an energy harvester module will require obtaining power from external environment where there is no guarantee of its availability [9]. Hence, it is always a wise decision to focus on energy optimization principle rather than looking for other non-sustainable alternatives for energy conservation in WSN. The study towards energy problems is very old and various standard protocols as well as schemes has been already evolved out [10]-[14]; however, there is no such scheme which has offered a proof that there is a long lasting sensor. Basically, a sensor is a very miniature device characterized by external battery as well as its components is characterized by low processing capability too. Even if a potential solution towards energy efficiency is designed, it is essential that it should be operationally supported by the processing components of a sensing device. Existing approaches has discussion of various energy conservation techniques but they are highly symptomatic in nature which deals with one set of problem only. Hence, in spite of large archives of investigation work towards energy issues in WSN, the problem is yet an open-end problem by large. Apart from this, it is also known that a sensor node is an integral part of Internet-of-Things (IoT) which offers further magnified connectivity of sensors [15]. IoT connects all nodes with common platform of internet and certain operating devices like switches and gateways [16]. The amount of traffic on IoT is extensively more compared to WSN and existing standards of energy conservation techniques are not directly executed over an IoT platform leading to another set of incompatibility. This problem of offering a communication bridge by using a translation services is now possible by using gateway in IoT. However, the question still is left open: How to optimize the energy in sensor nodes when connected in form of network? The answer to this question would possibly lead us towards evolving a novel energy optimization principle in future.

Therefore, this paper contributes towards offering a compact snapshot of strength and weakness of existing schemes which is claimed for offering energy optimization in WSN. The idea is to assist the research community to make a practical decision and derive a conclusive remark about the existing picture of different variants of energy optimization schemes in WSN. The organization of the proposed manuscript is as follows: Section II discusses about the WSN energy optimization scheme in existing times followed by discussion of existing approaches of energy optimization in Section III. Section IV highlights about existing trends of research approaches followed by significant highlights of open-end research problems in Section V. Finally, study findings of this paper are presented in Conclusion under Section VI. 


\section{INSIGHTS ON WSN ENERGY OPTIMIZATION}

This section discusses about all the necessary information associated with energy optimization in WSN. At present, there are three terms which looks more-or-less alike to each other with respect to energy e.g., energy efficiency, energy aware, and energy optimization. The first term energy efficiency is about implementation scheme which attempts to restore maximum amount of possible energy by adopting different methodologies. However, the final outcome of energy consumption could be still high, but this is the most practical plan and widely used. The second term energy aware is based on implementation techniques using budgeted amounts of resources in presence of test environment. Such techniques are good for assessing smaller scale of implementation or when the test environment is practically equivalent to real-environment of implementation. The third term energy optimization is associated with those techniques where the target is to reduce energy consumption without adding or utilizing more number of resources. There has been some number of studies towards energy optimization in WSN and still it is an ongoing arena of research in order to yet come out with some fail-proof solution. Prior to understanding the energy optimization, it is necessary to review various essential points associated with this. They are briefed as below:

\section{A. Reason for Energy Depletion in WSN}

A modern architecture of a sensor node basically consists of a power unit and power harvester [17]. There are various components within a power unit i.e., sensing units, computing units, communication unit, and power unit. All this units have different ranges of energy consumption. All the existing and ongoing research studies towards energy management system as well as optimization is carried out over the block of power management and energy prediction which resides within a computing unit of sensor hardware architecture as shown in Fig. 1.

It will mean that other units (power, sensing, and communication) are ignored while modelling existing solution. Hence, existing models are not found to consider the complete hardware perspective while modelling solution towards energy problems in WSN and hence the problems still exit todays.

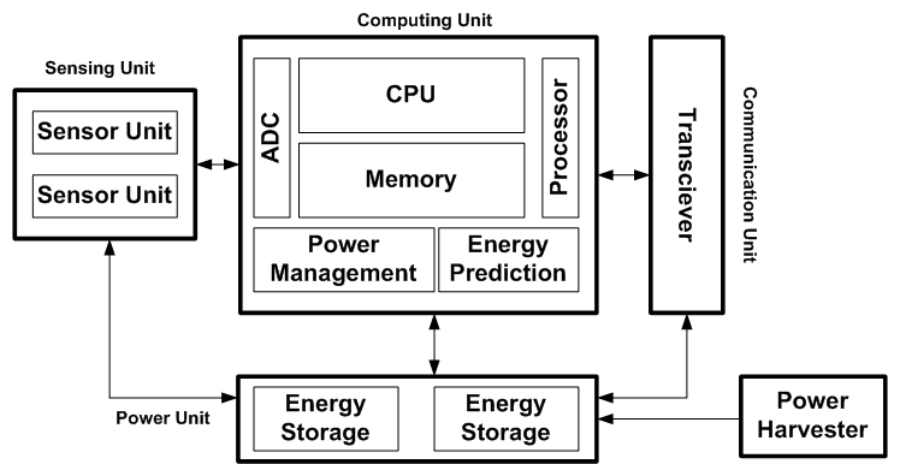

Fig. 1. Hardware Architecture of Sensor node in WSN [17].
However, the good news is that existing research work towards energy problems has made some potential discover towards exploring prominent reason for energy depletion in WSN as follows:

- Energy Conservation Problems: This problem is associated with predicting data, compressing higher ranges of aggregated data, reduction of data size, and optimizing the radio. The significant problem is that none of the above-mentioned attributes has been ever combinely studied for solving energy problems [17].

- Energy Transfers Problems: This kind of problem is mainly linked with power units of a sensor and therefore it is essential to consider. Various problems associated with charging techniques, energy transfer techniques, its associated tools, integrated data and energy transmission are essential attributes of an upcoming investigation in WSN, which is still under the roof of research and development [17].

- Energy Harvesting Problems: Currently, all the sensor node that is meant for deploying in critical environment are equipped with harvester node. At present, there are various ongoing and completed research in this perspective [17]; however, the addressed issues are still associated with source of energy to be harvested, energy storage, and performing neutral operation on energy.

- Addressing Routing Issues: At present, there are good number of research publication where routing [18] and clustering [19] has been used for achieving energy conservation in WSN. However, this scheme majorly doesn't consider the complete attributes that causes energy depletion. Hence, the effectiveness of such solution is only partially reasonable and applicable in real world environment.

\section{B. Existing Studies on Energy Optimization}

There are various studies that have been carried out towards energy optimization schemes by various means. Different nonlinear optimization issues have been discussed by AsoreyCacheda et al. [20] while multi-objective optimization schemes are reviewed by Fei et al. [21]. According to Cao et al. [22], there is a potential contribution of swarm intelligence-based optimization scheme applicable in case of involvement of mobility in sensor network. The work carried out by Diab et al. [23] discussed various data transmission scheme in WSN integrated with cognitive network emphasizing on energy constraints. The study carried out by Srinidhi et al. [24] have discussed about network optimization associated with IoT. The most recent study carried out by Khisa and Moh [25] has presented review of updated data transmission scheme towards energy efficiency associated with underwater WSN applications. Discussion presented by Williams et al. [26] has presented updated schemes of energy harvesting schemes in WSN. Therefore, there are various existing approaches towards addressing energy optimization under different methodologies. However, the extent of successful optimization associated with energy is yet not quantified till date. 


\section{EXISTING APPROACHES OF ENERGY OPTIMIZATION}

This section briefs about different approaches targeting towards optimizing energy. The term optimization experimentally refers to accomplishing the target aim of controlling every factor and reason to reduce energy efficiency without inclusion of very large or significant number of resources involved in it. However, it is yet to investigate the degree of optimization used in the adopted approaches, its degree of solution associated with problems, its beneficial aspect as well as its limiting factor. The proposed investigation witnesses different methods towards an objective of solving energy problems in WSN and hence, they have been clustered into six different approaches as discussed below:

\section{A. Game-based Approach}

From the theoretical aspect, game theory is basically known to assists in making decision for a given number of confusing choices of strategies [27]. Owing to the potential of carrying out decision, game theory is used in carrying out decision towards data forwarding in WSN [28]. The sensor node can be assumed as a player in game theory who is required to opt for choosing a route for data transmission towards base station (in single hop) or to neighboring cluster head (in multi-hop). At present, there is a slowly increasing adoption towards using game theory; however, the target is again a scattered one. The work carried out by Chowdhury et al. [29] targets towards two problems i.e., energy depletion and congestion in WSN emphasizing over low-end sensor node deployment. The author has designed non-cooperative game in order to optimize the process of improving data forwarding rate with an idea of resisting bottleneck condition in WSN. Game theory is also witnessed to be used in improving clustering approach as seen in work of Lin et al. [30] where a game model is designed using Nash Equilibrium point selecting dual cluster heads. This work uses energy and distance to carry out the process of optimizing cluster head. Apart from this, it is also known that Software-Defined Network (SDN) significantly improves network performance of WSN. This fact is reported to be further improved upon by Peizhe et al. [31] where game theory is used by mapping the SDN and WSN. Different from all above-mentioned work, Shahrokhzadeh and Dehghan [32] has presented a unique study where coverage performance is improved for WSN with capability to sense visual signals. A unique distributed game model is constructed which bridges the gap between energy depletion and quality of network coverage of visual sensor node. Another unique approach of game modelling is carried out by Zayene et al. [33]. The solution is for addressing the problems associated with cooperative exchange of data. This model is constructed on the basis of consumed energy and completion time where a unique merge and split algorithm is constructed along with network coding which can perform instantaneous decoding capabilities. A coalition game model is developed emphasizing over achieving energy efficiency assessed over a test bed of WSN with mobility aspect being considered. Hence, game theory has offered some unique techniques in order to perform routing which directly or indirectly also controls energy efficiency in WSN.

\section{B. Machine Learning Approach}

The evolution of Machine Learning (ML) approaches dates back more than two decades old while its implementation witnesses a potential hike in various forms of applications and services. Powered by the potential of artificial intelligence, ML approach offers significant capability to solve complex problem without any instruction from humans [AR]. At present, ML approaches are widely used in networking in order to enhance its management, analytical operation, and security [AR]. Different numbers of computational models are used in order to embody ML approach. In the area of WSN, ML has been used for multiple purposes which directly or indirectly is linked with routing operation. Basically, ML approaches are classified into supervised approach and unsupervised learning approach (Fig. 2).

- Supervised Learning Approach: Supervised learning approach is further categorized as classification and regression-based approaches. From classification perspective, there are four variants viz. Support Vector Machine (SVM), discriminant analysis, naïve bayes, and nearest neighbor. In recent studies, SVM is reported to be used for improving the accuracy of the sensor readings in WSN (Jeong et al. [34]). The usage of SVM is also witnessed in work of Li et al. [35] where the idea is mainly to offer fault detection in WSN considering temperature and acceleration. The next role of classification approach is played by discriminant analysis where it is reported to be used for formulating dynamic adjustment policy toward monitoring multiple sinks in WSN for data aggregation (Chen et al. [36]). Another variant of classification scheme under supervised learning policy is Naïve Bayes which performs probability-based classification (Chu et al. [37]). The work carried out by Barnawi and Keshta [38] have carried out a study where Naïve Bayes is studied alongside with multi-layered perceptron and SVM. The outcome of this study is not in favor of Naïve Bayes as better form of classification existing in current times. Finally, the nearest neighboring approach is used in supervised learning approach in WSN. The work carried out by $\mathrm{Fu}$ et al. [39] have used nearest neighboring approach for performing query processing in WSN with an idea of optimizing latency and energy involved. The work carried out by Marchang and Tripathi [40] have focused on crowd sensing particularly on spatial and temporal perspective. From regression-based supervised learning approach, there are following approaches seen to be used in WSN i.e., Linear Regression (LR), Support Vector Regression (SVR), Ensemble method, Decision Trees, and Neural Network. The first form of the supervised regression scheme is Linear Regression scheme. The work carried out by Yilmaz et al. [41] has carried out study of WSN with respect to achieve the accuracy of target along with stopping criterion formulation. Upadhyay et al. [42] have implemented Gaussian Regression of nonlinear form in order to form an effective clock synchronization. The scheme uses adaptive modelling with outcome of lower error rate in time synchronization. The next technique of regression is 
Support Vector Regression (SVR) which is meant for performing analysis of data with respect to classification and regression. The work carried out by Shamshirband et al. [43] where SVR is combined with Kalman filtering in order to carry out data fusion in WSN. The study outcome shows that SVR performs well with Radial Basis Function better than other SVR approaches with polynomial kernel approach. The work carried out by Guo et al. [44] have used SVR for the purpose of predicting the energy required for forwarding data in WSN of distributed form. The outcome shows SVR performing better with random forest regression. The next method is Ensemble learning which is another frequently used mechanism in regression-based supervised learning scheme. This method makes use of different learning models in order to achieve its optimization target. Most recently, the work is being carried out by Alotaibi [45] have used this technique for detecting the mode of sensor-based transportation application. The study outcome shows different accuracy score for different dataset. Study towards optimizing sensory array was witnessed in work of Wijaya [46] where ensemble method is used for monitoring the quality of food. The next prominent process of regression in supervised learning method is decision tree. The study carried out by Rout et al. [47] and Zheng et al. [48] have used decision tree for addressing energy conservation problems and recognition problems in WSN. The final form of regression-based supervised learning scheme is neural network, which is also one of the most frequently deployed schemes in wireless networks. Neural network implementation has been seen in the study carried out by Lin et al. [49], Wu et al. [50], and Mukherjee et al. [51] addressing the problems associated with energy consumption, energy harvesting, and identification of cluster head respectively.

- Unsupervised Learning approach: This approach basically carries out clustering operation which are further classified into Fuzzy C-Means, Gaussian Mixture, Neural Network, and Hidden Markov Model. Fuzzy-C Means (FCM) clustering offers the benefit of more tangible unsupervised learning by offering more data points for analysis. The work of Cheng et al. [52] has used FCM approach towards location quantification using received signal strength indicator in WSN. Further usage of FCM was witnessed in study of Fei et al. [53] emphasizing over clustering operation towards energy efficiency. The second type of unsupervised learning scheme is Gaussian Mixture Model (GMM) which is a potential clustering algorithm. A unique usage of GMM was witnessed in work of Shi and Feng [54] where the target is to retain maximum signal in WSN. On the other hand, GMM was also witnessed in work of Eguchi et al. [55] which carry estimation of joint angle. Discussion of recent studies in neural network is carried out in previous approach. The final model of unsupervised clustering technique is Hidden Markov Model (HMM). The work carried out by Liu et al. [56] have used HMM for training occupancy sensing while the work of Xu \& Wang [57] have used HMM for meteorology factor identification.

Fig. 2 highlights the taxonomies of existing ML approaches. Proliferated usage of ML approaches in WSN is seen in work of Kim et al. [58]. Apart from the above discussed frequently used ML approaches, there are various other approaches too viz. Deep reinforcement learning (Ashiquzzaman et al. [59], Ke et al. [60], Nguyen et al. [61]), deep neural network with sparse autoencoder (Ayinde [62]), opportunistic routing (Dinh et al. [63]), energy saving using simulated annealing (Kang et al. [64]), and energy harvesting using artificial neural network combined with linear regression (Kwan et al. [65]), Hence, it can be said that there are various dedicated research attempt towards using ML approach over WSN; however, not all the approaches are found to directly address energy problems in WSN.

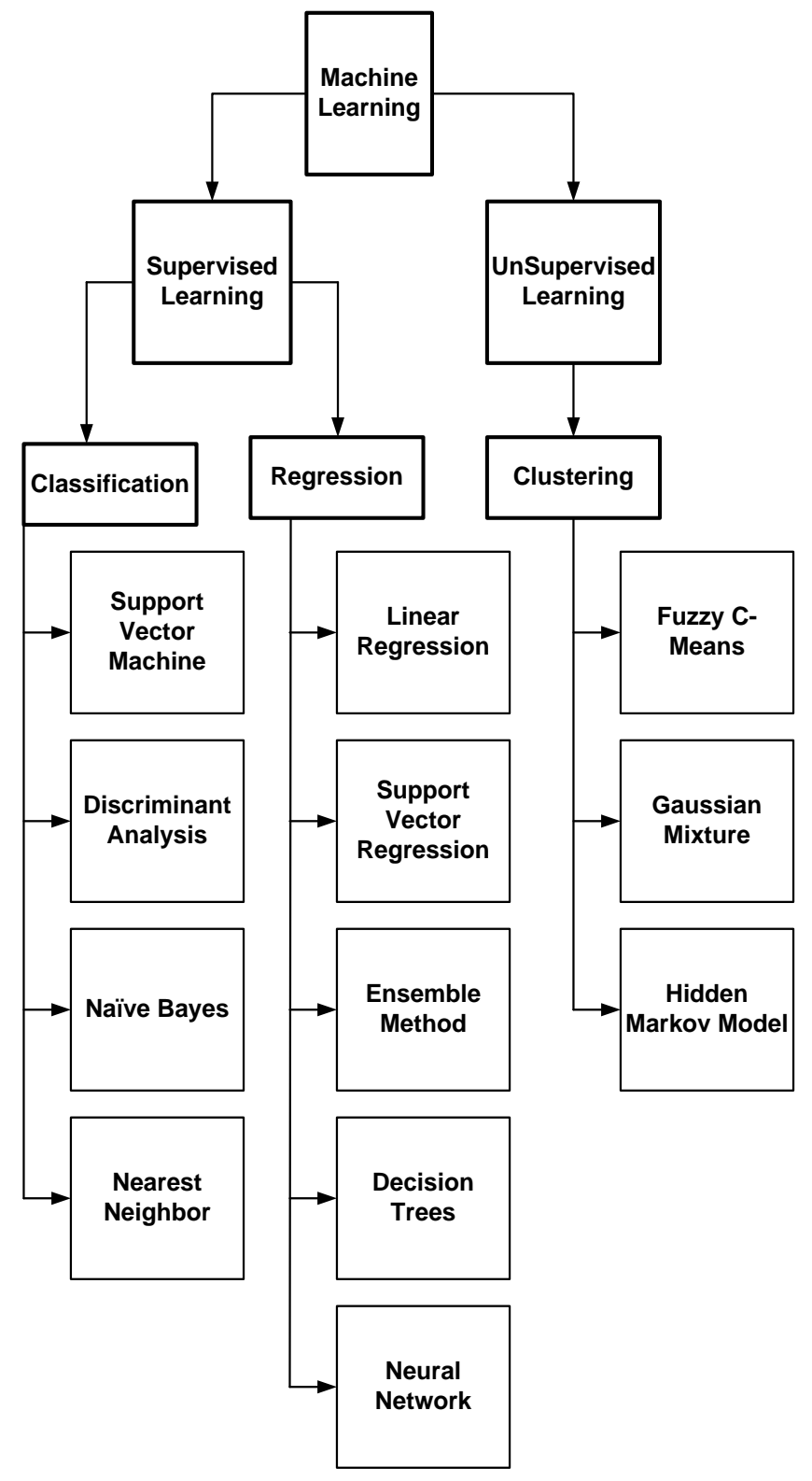

Fig. 2. Taxonomy of ML based Approach in WSN (Need Amendment). 


\section{Scheduling Approach}

Scheduling based approaches mainly make use of making a proper routine along with provisioning of resources in order to ensure accomplishment of pre-defined target. Provisioning scheme assists in optimal deployment of sensor as witnessed in study of Du et al. [66]. Scheduling scheme is also reported to save energy depletion along with secure communication in WSN (Feng et al. [67]) using probability-based modelling. Scheduling approach is also applied for estimating the state of the remoteness on the basis of stochastic event considering the case study of WSN with cognitive radio networks (Huang et al. [68]). It was also noticed that there is a significant improvement in system performance when allocation of different attributes of resource is considered in scheduling process. Such direction of work is carried out by Li et al. [69] towards tracking multiple targets; however, it lacks optimization. Focus on optimizing the scheduling from temporal aspects is seen in work carried out by Liu et al. [70] which is basically meant for IoT networks by improving the power allocation ratio. A different form of study has been carried out by Shi et al. [71] in order to address the problems associated with convergecast scheduling. The idea of this work is mainly to increase the number of concurrent transmissions in WSN considering the constraints of integer programming. The work of Wan et al. [72] has presented discussion about sleep scheduling approach in WSN for conserving energy. The study has used fuzzy concept in order to perform organization of sensors along with aggregation of data on the basis of similarity matrix. Wang et al. [73] have carried out study towards deterministic scheduling for controlling an effective transmission delay. The study implements a scheduling rule on the basis of classified link conflict and initial reduced extent of conflict. The work carried out by Yang et al. [74] has presented a scheduling policy using unique power allocation scheme in WSN. Another scheduling scheme using distributed Multiple Input Multiple Output (MIMO) has been implemented by Zhang et al. [75] emphasizing over allocation of resource and scheduling of device for the purpose of detection and tracking in WSN.

\section{Harvesting Approach}

Energy harvesting technique is strongly linked with energy optimization scheme in WSN. Using varied sources of energy (magnetic, vibration, temperature, solar, etc.), a harvester node stores energy ensuring to deliver a satisfactory backup for the energy supply demanded by the specific sensor node. Hence, node using harvesting module should have better harvesting capabilities. At present, there are existing research studies towards energy harvesting techniques in WSN are i) using reinforcement learning (Aoudia et al. [76]), duty-cycle based modelling (Chew et al. [77]), opportunistic routing with cooperative charging (Hu et al. [78]), intra-vehicular communication based energy harvesting (Koca et al. [79]), energy aware interface design with vibration-based energy harvesting (Ruan et al. [80]), polynomial time-scheduling for solar energy harvesting (Wang et al. [81]), multi-objective optimization based hybrid energy harvesting (Xiong et al. [82]), and optimized data buffer scheme (Zhu et al. [83]).

\section{E. Cross Layer Approach}

From the viewpoint of protocol layer, both hierarchical and cross-layer approach is meant for incorporating optimized performance growth in WSN. However, hierarchical architectures suffer from issues e.g., signal fading, interference between users, access conflicts. However, proper maximization of the limited resources in protocol stack is not possible in hierarchical design, which is possible using cross-layer approach. By using cross-layer approach, it is possible for different layers to interact with each other for exchange of information. This phenomenon is utilized for solving different issues in WSN. From bottom to top, the protocol stack of WSN consists of IEEE 802.15.4 layer, which has data link layer and physical layer, network layer, transmission control layer, and application support layer. At present, there are various crosslayer based approach presented for improving performance on various aspects in WSN. However, there exists specifically only few approach associated with respect to energy aspect using following cross-layer based methodologies viz. routing reliability using time-based factor for energy (Abbasi et al. [84]), improved geographic routing for green efficiency in IoT with Markov queuing model (Hasan et al. [85]), maximizing network lifetime using cross layer (Hermann [86]), MAC scheduling with adaptiveness (Ngo et al. [87]), optimization of cross-layer for shortest and multipath routing (Shimly et al. [88]), optimizing data aggregation and choosing cluster head using particle swarm optimization using cross layer approach (Sun et al. [89]), optimizing energy-efficient routing for conventional LEACH protocol using cross layer (Zhang et al. [90]). Majority of the existing approaches of cross layer in WSN make use of network management layer which consist of physical layer, data link layer, network layer, and transmission control layer. They are meant for serving remote management, mobility control, network security, quality of service supportability, energy management, and topology control.

\section{F. Swarm Intelligence Approach}

This approach is designed on the basis of decentralized behaviour of self-organized system. Theoretically, swarm intelligence falls under the category of population-based search approach of metaheuristics method in optimization technique. It comes within approximated combinatorial method. They are designed on the basis of cognitive behaviour of certain biologically inspired entity e.g., ant, honeybee, firefly, frog, fish, cat, dolphin, etc. The studies that has used swarm intelligence linking with energy efficiency are as follows: Gray-wolf optimization (Arafat et al. [91]), Bat algorithm (Cao et al. [92]), flocking control scheme using swarm intelligence (Dai et al. [93]), firefly mating optimization (Faheem et al. [94]), fish algorithm with k-means clustering (Feng et al. [95]), multi-swarm optimization (Hasan et al.[96]), Harris' Hawk optimization (Houssein et al. [97]), particle swarm optimization (Mukherjee et al. [98]), Chicken swarm optimization (Osamy et al. [99]), reinforcement learning with swarm intelligence (Wei et al. [100]). However, different approaches have their own structure of working which is implemented on WSN on different targets of optimization towards energy efficiency. Table I summarizes the existing reviews. 
TABLE I. SUMMARY OF EXISTING OPTIMIZATION APPROACHES IN WSN

\begin{tabular}{|c|c|c|c|c|}
\hline Authors & Problems & Solution & Advantage & Limitation \\
\hline Chowdhury et al. [29] & $\begin{array}{l}\text {-energy depletion } \\
\text {-congestion }\end{array}$ & $\begin{array}{l}\text { Non-cooperative game, } \\
\text { optimizing data transmission } \\
\text { rate }\end{array}$ & $\begin{array}{l}\text { Better energy and delay } \\
\text { control }\end{array}$ & $\begin{array}{l}\text { Not applicable for } \\
\text { heterogeneous network }\end{array}$ \\
\hline Lin et al. [30] & Optimizing clustering & Dual cluster head selection & $\begin{array}{l}\text { Increase span of network } \\
\text { lifetime }\end{array}$ & $\begin{array}{l}\text { Doesn't deal with data } \\
\text { complexity and not applicable } \\
\text { for sparse network. }\end{array}$ \\
\hline Peizhe et al. [31] & Energy completion & SDN with game model & $\begin{array}{l}\text { A synced communication } \\
\text { between controller and sensor } \\
\text { node }\end{array}$ & $\begin{array}{l}\text { Doesn’t support dynamic } \\
\text { environment }\end{array}$ \\
\hline $\begin{array}{l}\text { Shahrokhzadeh and Dehghan } \\
\text { [32] }\end{array}$ & Improving coverage & $\begin{array}{l}\text { Learning algorithm on the } \\
\text { basis of pay-off. }\end{array}$ & Improve network lifetime & $\begin{array}{l}\text { not applicable for large } \\
\text { dynamic network. }\end{array}$ \\
\hline Zayene et al. [33] & Energy depletion & Coalition game model & Improve scalability & $\begin{array}{l}\text { Involves computational } \\
\text { complexity owing to highly } \\
\text { iterative operation. }\end{array}$ \\
\hline Jeong et al. [34], Li et al. [35] & Assessing reading accuracy & Support Vector Machine & Satisfactory accuracy & $\begin{array}{l}\text { Doesn't contribute towards } \\
\text { energy efficiency directly. }\end{array}$ \\
\hline Chen et al. [36] & Multi-sink monitoring & Discriminant Analysis & Good energy conservation & $\begin{array}{l}\text { Unable to differentiate data } \\
\text { acquisition process }\end{array}$ \\
\hline Barnawi and Keshta [38] & Comparative study & Naïve Bayes & Simpler comparison & $\begin{array}{l}\text { Naïve Bayes not found to be } \\
\text { an effective }\end{array}$ \\
\hline Chu et al. [37] & Classification of data fault & Naïve Bayes & $\begin{array}{l}\text { Higher accuracy in fault } \\
\text { detection }\end{array}$ & $\begin{array}{l}\text { Dynamic faults not accessed } \\
\text { in the study }\end{array}$ \\
\hline Fu et al. [39], Marchang [40] & $\begin{array}{l}\text { Query processing, crowd } \\
\text { sensing }\end{array}$ & Nearest neighboring & $\begin{array}{l}\text { Reduced resource } \\
\text { consumption }\end{array}$ & $\begin{array}{l}\text { Not applicable for } \\
\text { heterogeneous network }\end{array}$ \\
\hline Yilmaz et al. [41] & $\begin{array}{l}\text { Sequential estimation, } \\
\text { decentralized }\end{array}$ & Linear Regression & Effective stopping time & $\begin{array}{l}\text { Doesn't emphasize on peak } \\
\text { traffic condition }\end{array}$ \\
\hline Upadhyay et al. [42] & Clock synchronization & $\begin{array}{l}\text { Non-linear gaussian } \\
\text { regression }\end{array}$ & Reduced error & $\begin{array}{l}\text { Applicable on static } \\
\text { environment only }\end{array}$ \\
\hline Shamshirband et al. [43] & Comparison analysis of SVR & Kalman filtering + SVR & Higher precision & $\begin{array}{l}\text { Doesn't evaluate energy } \\
\text { efficiency }\end{array}$ \\
\hline Guo et al. [44] & Energy prediction & $\begin{array}{l}\text { SVR with random forest, } \\
\text { gradient boosting regression }\end{array}$ & Higher precision & $\begin{array}{l}\text { Doesn't evaluate energy } \\
\text { efficiency }\end{array}$ \\
\hline Alotaibi [45] & $\begin{array}{l}\text { Detecting mode of } \\
\text { transportation }\end{array}$ & Ensemble Learning & Satisfactory accuracy & $\begin{array}{l}\text { Doesn't deal with scalability } \\
\text { performance }\end{array}$ \\
\hline Wijaya [46] & Optimizing sensor array & Ensemble Learning & Higher accuracy & $\begin{array}{l}\text { Limited to static and low } \\
\text { scale application }\end{array}$ \\
\hline Rout et al. [47] & $\begin{array}{l}\text { Finding optimal switching } \\
\text { policy }\end{array}$ & Decision Tree, Markov & Satisfactory energy control & Absence of benchmarking \\
\hline Zheng et al. [48] & Recognition of target & Decision Tree & $\begin{array}{l}\text { Effective benchmarking } \\
\text { outcomes }\end{array}$ & $\begin{array}{l}\text { Not applicable for } \\
\text { heterogeneous and massive } \\
\text { data }\end{array}$ \\
\hline Lin et al. [49] & $\begin{array}{l}\text { Estimation of energy } \\
\text { depletion }\end{array}$ & $\begin{array}{l}\text { Neural Network (Radial basis, } \\
\text { generalized regression) }\end{array}$ & Lower energy consumption & $\begin{array}{l}\text { Lower scope of test } \\
\text { evaluation }\end{array}$ \\
\hline Wu et al. [50] & Energy harvesting & Neural Network & Higher learning efficiency & $\begin{array}{l}\text { Doesn't evaluate energy } \\
\text { efficiency }\end{array}$ \\
\hline Mukherjee et al. [51] & Identification of cluster head & $\begin{array}{l}\text { Neural Network (Back } \\
\text { propagation) }\end{array}$ & $\begin{array}{l}\text { Better control of energy } \\
\text { consumption }\end{array}$ & $\begin{array}{l}\text { Computational complexity } \\
\text { not analyzed. }\end{array}$ \\
\hline Cheng et al. [52] & Location Quantification & FCM & Simplified voting process & $\begin{array}{l}\text { The study is carried out in } \\
\text { low-end application } \\
\text { environment. }\end{array}$ \\
\hline Fei et al. [53] & Clustering & FCM & Energy efficient & $\begin{array}{l}\text { Leads to computational } \\
\text { complexity due to higher } \\
\text { iteration. }\end{array}$ \\
\hline Shi and Feng [54] & Signal restoration & GMM & $\begin{array}{l}\text { High quality of signal } \\
\text { preservation }\end{array}$ & $\begin{array}{l}\text { Doesn't address energy } \\
\text { optimization }\end{array}$ \\
\hline Eguchi et al. [55] & Estimation of joint angle & GMM & Higher accuracy & $\begin{array}{l}\text { Doesn't address energy } \\
\text { optimization }\end{array}$ \\
\hline Liu et al. [56] & $\begin{array}{l}\text { Training for accuracy in } \\
\text { Occupancy sensing }\end{array}$ & HMM & Improve sensing time & $\begin{array}{l}\text { Not applicable for } \\
\text { heterogeneous WSN }\end{array}$ \\
\hline Xu et al. [57] & $\begin{array}{l}\text { Quantification of fine } \\
\text { particulate }\end{array}$ & HMM & Simplified operation & Absence of benchmarking \\
\hline Du et al. [66] & Node placement & $\begin{array}{l}\text { Scheduling using wireless } \\
\text { energy transfer }\end{array}$ & Optimizes node deployment & Absence of benchmarking \\
\hline Feng et al. [67] & Energy saving & Sleep scheduling & $\begin{array}{l}\text { Energy saving performance is } \\
\text { good }\end{array}$ & $\begin{array}{l}\text { Not applicable for } \\
\text { heterogeneous network }\end{array}$ \\
\hline Huang et al. [68] & Estimating remote state & $\begin{array}{l}\text { Open-close loop scheduling } \\
\text { of stochastic events }\end{array}$ & Reduces communication rate & $\begin{array}{l}\text { Doesn't discuss its impact on } \\
\text { energy consumption }\end{array}$ \\
\hline Li et al. [69] & Tracking multi target & $\begin{array}{l}\text { Resource allocation, sensor } \\
\text { scheduling }\end{array}$ & Effective resource saving & $\begin{array}{l}\text { Involves computational } \\
\text { overhead. }\end{array}$ \\
\hline
\end{tabular}




\begin{tabular}{|c|c|c|c|c|}
\hline Liu et al. [70] & $\begin{array}{l}\text { Increasing data transmission } \\
\text { rate }\end{array}$ & Time scheduling & $\begin{array}{l}\text { Effective analysis of outage } \\
\text { probability }\end{array}$ & $\begin{array}{l}\text { Complete power attributes for } \\
\text { device not considered }\end{array}$ \\
\hline Shi et al. [71] & $\begin{array}{l}\text { Maximize concurrent } \\
\text { transmission }\end{array}$ & Convergent scheduling & Reduces latency & $\begin{array}{l}\text { Doesn't evaluate energy } \\
\text { extensively }\end{array}$ \\
\hline Wan et al. [72] & Energy efficiency & Sleep scheduling & Ensure data accuracy & Not assessed for scalability \\
\hline Wang et al. [73] & $\begin{array}{l}\text { Reduction of transmission } \\
\text { delay }\end{array}$ & $\begin{array}{l}\text { Scheduling based on } \\
\text { classified link conflict }\end{array}$ & Low complexity & Low scale deployment \\
\hline Yang et al. [74] & Distributed filtering & $\begin{array}{l}\text { Power allocation, } \\
\text { experimental }\end{array}$ & Improves estimation accuracy & Low scale deployment \\
\hline Zhang et al. [75] & $\begin{array}{l}\text { Detection and tracking of } \\
\text { target }\end{array}$ & $\begin{array}{l}\text { Allocation of resource, } \\
\text { distributed MIMO }\end{array}$ & Lower computational burden & $\begin{array}{l}\text { Iterative scheme leading to } \\
\text { lowering of network lifetime } \\
\text { in long round. }\end{array}$ \\
\hline Aoudia et al. [76] & Energy harvesting & Reinforcement learning & $\begin{array}{l}\text { Significant increase in } \\
\text { average packet rate }\end{array}$ & $\begin{array}{l}\text { Doesn't address bottleneck } \\
\text { condition in traffic }\end{array}$ \\
\hline Chew et al. [77] & $\begin{array}{l}\text { Energy harvesting for TSCH } \\
\text { nodes }\end{array}$ & $\begin{array}{l}\text { Network joining using duty- } \\
\text { cycle }\end{array}$ & Simplified design architecture & Absence of benchmarking \\
\hline Hu et al. [78] & Environmental changes & $\begin{array}{l}\text { Integrating energy transfer } \\
\text { approach with energy } \\
\text { harvesting, opportunistic } \\
\text { routing }\end{array}$ & Enhanced network lifetime & $\begin{array}{l}\text { Not applicable for dynamic } \\
\text { and large-scale network }\end{array}$ \\
\hline Koca et al. [79] & $\begin{array}{l}\text { Feasibility analysis of energy } \\
\text { harvesting in vehicles }\end{array}$ & $\begin{array}{l}\text { Intra-vehicular } \\
\text { communication }\end{array}$ & $\begin{array}{l}\text { Effective transmission } \\
\text { performance }\end{array}$ & $\begin{array}{l}\text { Leads to computational } \\
\text { complexity }\end{array}$ \\
\hline Ruan et al. [80] & Energy harvesting & Harvesting using Vibration & Energy aware modelling & Doesn’t ensure scalability \\
\hline Wang et al. [81] & Wireless charging & $\begin{array}{l}\text { Harvesting using Solar, } \\
\text { polynomial time scheduling }\end{array}$ & $\begin{array}{l}\text { Reduce energy consumption } \\
\text { and cost of vehicle moving }\end{array}$ & $\begin{array}{l}\text { Time of wireless charging not } \\
\text { sufficient enough in case of } \\
\text { large traffic scenario }\end{array}$ \\
\hline Xiong et al. [82] & Energy harvesting & $\begin{array}{l}\text { Bidirectional local search, } \\
\text { particle swarm optimization } \\
\text { with multiple objectives }\end{array}$ & Prolonged network lifetime & Not scalable for large traffic \\
\hline Zhu et al. [83] & $\begin{array}{l}\text { Maximize throughput during } \\
\text { harvesting }\end{array}$ & $\begin{array}{l}\text { Data buffer, super-frame } \\
\text { optimization, stochastic } \\
\text { modelling }\end{array}$ & Improved throughput & $\begin{array}{l}\text { Specific to device with IEEE } \\
802.15 .4 \text { standard only }\end{array}$ \\
\hline Abbasi et al. [84] & Communication reliability & Cross-layer based routing & Better quality of service & $\begin{array}{l}\text { Lacks optimization of } \\
\text { resources }\end{array}$ \\
\hline Hasan et al. [85] & $\begin{array}{l}\text { Essential of cooperative } \\
\text { demands in multihop } \\
\text { communication }\end{array}$ & $\begin{array}{l}\text { Cross-layer based queuing } \\
\text { model, geographic switching }\end{array}$ & More green efficiency & $\begin{array}{l}\text { Doesn't consider hardware- } \\
\text { based factors for switching }\end{array}$ \\
\hline Herrmann et al. [86] & Maximizing network lifetime & Lifetime optimization & $\begin{array}{l}\text { Significant energy depletion } \\
\text { control }\end{array}$ & Not scalable for large traffic \\
\hline Ngo et al. [87] & $\begin{array}{l}\text { Adaptive scheduling in body } \\
\text { sensor network }\end{array}$ & Cross layer, MAC scheduling & Better quality of service & Absence of benchmarking \\
\hline Shimly et al. [88] & Any-to-any routing & Cross layer optimization & Reduce retransmission & $\begin{array}{l}\text { Possess overhead when } \\
\text { exposed to dynamic traffic }\end{array}$ \\
\hline Sun et al. [89] & Clustering & Cross-layer sensing & $\begin{array}{l}\text { Optimize aggregation } \\
\text { efficiency }\end{array}$ & Highly iterative process \\
\hline Zhang et al. [90] & Clustering efficiency & $\begin{array}{l}\text { Cross layer optimization of } \\
\text { LEACH protocol }\end{array}$ & Reduce cluster computation & $\begin{array}{l}\text { Not analyzed with dynamic } \\
\text { traffic system }\end{array}$ \\
\hline Arafat et al. [91] & Clustering, localization & $\begin{array}{l}\text { Compressive sensing based } \\
\text { on gray-wolf optimization }\end{array}$ & Efficient routing tree & Involves cost and complexity \\
\hline Cao et al. [92] & Data fusion & Bat algorithm & Improve network lifetime & $\begin{array}{l}\text { Cannot handle bottleneck } \\
\text { condition }\end{array}$ \\
\hline Dai et al. [93] & $\begin{array}{l}\text { Decision making for } \\
\text { autonomous vehicular } \\
\text { movement }\end{array}$ & $\begin{array}{l}\text { Swarm intelligence, } \\
\text { experimental }\end{array}$ & Low energy consumption & Doesn’t ensure data quality \\
\hline Faheem et al. [94] & Energy-efficient routing & Firefly mating optimization & $\begin{array}{l}\text { Ensure satisfactory quality of } \\
\text { service }\end{array}$ & $\begin{array}{l}\text { Residual energy could be } \\
\text { optimized more }\end{array}$ \\
\hline Feng et al. [95] & $\begin{array}{l}\text { Optimizing Network } \\
\text { Coverage }\end{array}$ & $\begin{array}{l}\text { Fish swarm algorithm, K- } \\
\text { means algorithm }\end{array}$ & Good energy saving & Tested over small network \\
\hline Hasan et al. [96] & $\begin{array}{l}\text { Meeting Quality of service for } \\
\text { Industrial IoT }\end{array}$ & Particle Swarm Optimization & Satisfactory quality of service & Tested over small network \\
\hline Houssein et al. [97] & Sink node placement & Harris Hawk Optimization & Reduced energy consumption & $\begin{array}{l}\text { Throughput is dependent on } \\
\text { node position }\end{array}$ \\
\hline Mukherjee et al. [98] & Resource allocation & $\begin{array}{l}\text { Adaptive Particle Swarm } \\
\text { Optimization }\end{array}$ & $\begin{array}{l}\text { Faster response time and } \\
\text { reduced energy }\end{array}$ & $\begin{array}{l}\text { Computationally complex } \\
\text { process }\end{array}$ \\
\hline Osamy et al. [99] & Clustering & Chicken Swarm Optimization & Enhanced network lifetime & Static fitness function design \\
\hline Wei et al. [100] & Charging sensors & $\begin{array}{l}\text { Firefly algorithm, } \\
\text { Reinforcement learning }\end{array}$ & Better energy utilization & $\begin{array}{l}\text { Dynamic energy consumption } \\
\text { not evaluated. }\end{array}$ \\
\hline
\end{tabular}




\section{Current Research Trends}

The previous section has discussed about taxonomies of all the existing approaches that are frequently used for solving energy problems in WSN. However, all the solutions evolved up till date cannot be termed as optimized problems. There are different categories of research-based solution towards this purpose by different names; therefore, this paper contributes towards forming up a standard name of this approach in order to understand the current research trends. Fig. 3 highlights the number of different publications towards different types of research-based solution for addressing energy problems in WSN.

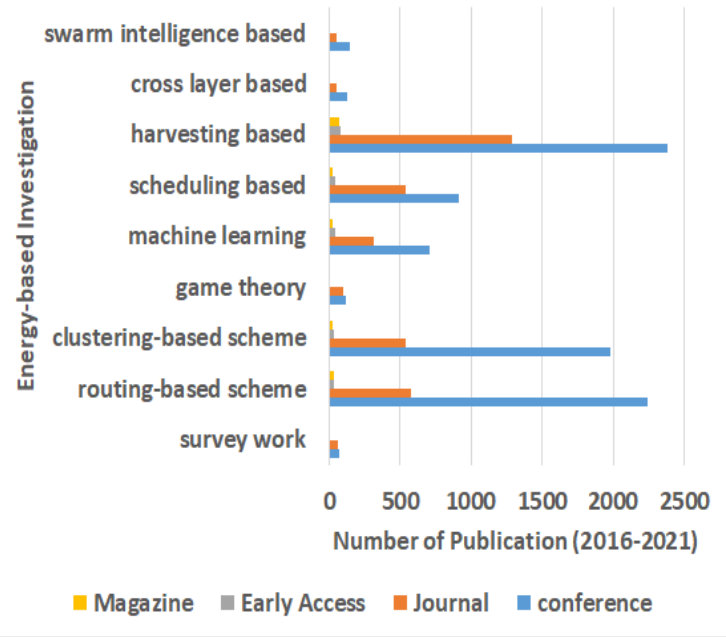

Fig. 3. Current Research Trend towards Energy in WSN.

Following are the findings of the research trends seen from the research-based manuscript published within the year of 2016-2021 as one of the contributions of proposed study being carried out:

- More insights towards research approaches are now evolving towards energy harvesting based approaches. Although, this is another level of future of using sensory application for network lifetime improvement but yet no robust standardization is yet obtained for harvesting models in WSN. It still has a long way to go.

- Routing-based and clustering-based approaches are the most frequently used approach that are being consistently adopted as core research problem since last decade. There are evolution of standard routing and clustering techniques known for energy efficiencies in WSN; but still, they do not encapsulate all the factors responsible for energy depletion.

- Scheduling-based and machine learning is next frequently adopted approach for energy efficiencies. Although, these approaches are proven fruitful towards energy conservation schemes in WSN but they are less inclined towards energy optimization.

- A unique observation is obtained from cross-layer based and game theory-based schemes as they are not yet found fruitful towards energy optimization. They are proven fruitful for solving other problems in WSN but not much in energy control and management.

- Adoption of swarm intelligence is still a novel approach towards energy optimization although they are proven effective for solving other problems in WSN. There is a need of more number of researches in order to consider this approach towards energy optimization.

\section{Open Research Challenges}

After reviewing the existing studies towards controlling energy problems in WSN, certain level of conclusive remarks has been formulated. The open research challenges associated with the achieving optimized energy in WSN are briefed as following:

- Mono-issue Modelling Practices: Majority of the discussed techniques is found to address a specific problem which is associated with energy. For an example, problems associated with routing and energy is considered without considering hardware inclusion or without considering clustering techniques. Such approaches cannot be considered as wholesome solution as other connected factors related to energy are often found ignored, irrespective of any techniques discussed in this study. Hence, majority of the modelling practices in current times considers addressing one problem of energy in WSN.

- Less Focus on Practical Modelling: It should be known that majority of the application of WSN is hypothetical and less practical from commercial and real-time aspect. Commercial practices in current times calls for using sensors in IoT or smart city development which has massive number of challenges and issues to be sorted out apart from energy problems. Apart from this, even if WSN domain is researched than there is a need to consider heterogeneity in protocol implementation, data quality, data complexities, environmental parameters affecting data transmission should be considered. Protocols of WSN are not applicable in IoT and protocols of IoT are not applicable in WSN. Hence gateway concept comes in features to bridge this incompatibility issues. This aspect of energy modelling is found extremely low in existing studies in WSN as well as in IoT towards energy challenges.

- Ambiguity in Defining Optimization: There are various manuscript with title or with discussing of using optimization principle to reduce energy. However, this is slightly far from reality as such papers are often found with a big gap between their theoretical implication and practical implication. The practical implication of optimization concept calls for an evidential proof of higher energy retention with lower involvement of resources (both hardware and software). It is also required to offer an evidence that optimized outcome is also scalable in nature for truly understanding its effectiveness. Further computational complexity is also required to be proven low, which is not found in existing. 
- Absence of Benchmarking during Analysis: Adoption of analytical test bed which has universal acceptance towards performance parameters, test values, initialized values of simulation parameters, involvement of standard and reliable energy computational, and involvement of multiple test cases is very much required. Existing studies towards energy optimization, although they are few in relevancy of implementation, are not found to be benchmarked.

\section{CONCLUSION}

Majority of existing as well as upcoming application of WSN demands a node to work for an extensive period of time unattended. This paper has discussed about various approaches which are found to address the problems associated with energy factor. There are large number of techniques in existing system towards energy problems in wireless sensor network, but there is a lack of compact study carried out towards discussion effectiveness of recent literatures. Hence, this paper contributes to review maximum number of significant implementation in energy problems in wireless sensor network and contributes to highlight the appropriate information associated with strength and weakness of existing studies. This findings will contribute towards research community to make a better decision towards evolving up with a necessary model to deal with identified problems and challenges in energy efficiency in sensory network. The contribution of this paper are in terms of essential findings as follows viz. i) unlike existing review studies or discussion paper, this paper makes a categorization of extensively used energy conservation approaches which are claimed to offer optimization; ii) there is highly scattered study towards addressing the challenges associated with energy optimization in WSN, in fact, there are extremely few existing approaches to offer evidence of energy optimization in WSN; iii) almost all the existing approaches are found to have limitations which is necessary to address in order to show practicality as well as adaptability of existing models; and iv) here is a need to evolve up with a novel mathematical model with multi-objective function and with less iterative scheme to offer benchmarked outcomes and cost effectively in data aggregation of WSN. Hence, developing an effective scheme for ensuring better lifetime in sensory application demands inclusion of various factors, e.g. states of nodes, types of links, scheduling scheme being used, inclusion of both hardware and software resources, as well as algorithm effectiveness. The lighter the algorithm operation, more is computational efficiency that will result in more extensive operation resulting in improved network lifetime.

Hence, the future work will be carried out in the direction of addressing the research gap discussed the proposed study. The future work should be initially designed to construct a formulation which considers all the practical constraints of WSN followed by developing a mathematical modelling of energy optimization. Essential check point should be given to ensure less iterative, faster computation, and higher data quality, and cost effectiveness along with scalability is ensured along with future modelling of energy optimization.

\section{REFERENCES}

[1] J. Liu, Z. Zhao, J. Ji and M. Hu, "Research and application of wireless sensor network technology in power transmission and distribution system," in Intelligent and Converged Networks, vol. 1, no. 2, pp. 199220, Sept. 2020, doi: 10.23919/ICN.2020.0016.

[2] S. Liao and Q. Zhang, "A Multiutility Framework With Application for Studying Tradeoff Between Utility and Lifetime in Wireless Sensor Networks," in IEEE Transactions on Vehicular Technology, vol. 64, no. 10, pp. 4701-4711, Oct. 2015, doi: 10.1109/TVT.2014.2372793.

[3] X. Yang, L. Wang and Z. Zhang, "Wireless Body Area Networks MAC Protocol For Energy Efficiency and Extending Lifetime," in IEEE Sensors Letters, vol. 2, no. 1, pp. 1-4, March 2018, Art no. 7500404, doi: 10.1109/LSENS.2018.2795566.

[4] J. Shi, M. Sha and Z. Yang, "Distributed Graph Routing and Scheduling for Industrial Wireless Sensor-Actuator Networks," in IEEE/ACM Transactions on Networking, vol. 27, no. 4, pp. 1669-1682, Aug. 2019, doi: 10.1109/TNET.2019.2925816.

[5] D. Sharma and A. P. Bhondekar, "Traffic and Energy Aware Routing for Heterogeneous Wireless Sensor Networks," in IEEE Communications Letters, vol. 22, no. 8, pp. 1608-1611, Aug. 2018, doi: 10.1109/LCOMM.2018.2841911.

[6] N. Aslam, K. Xia and M. U. Hadi, "Optimal Wireless Charging Inclusive of Intellectual Routing Based on SARSA Learning in Renewable Wireless Sensor Networks," in IEEE Sensors Journal, vol. 19, no. 18, pp. 8340-8351, 15 Sept.15, 2019, doi: 10.1109/JSEN.2019.2918865.

[7] H. Ko and S. Pack, "OB-DETA: Observation-based directional energy transmission algorithm in energy harvesting networks," in Journal of Communications and Networks, vol. 21, no. 2, pp. 168-176, April 2019, doi: 10.1109/JCN.2019.000015.

[8] T. Ruan, Z. J. Chew and M. Zhu, "Energy-Aware Approaches for Energy Harvesting Powered Wireless Sensor Nodes," in IEEE Sensors Journal, vol. 17, no. 7, pp. 2165-2173, 1 April1, 2017, doi: 10.1109/JSEN.2017.2665680.

[9] D. Newell and M. Duffy, "Review of Power Conversion and Energy Management for Low-Power, Low-Voltage Energy Harvesting Powered Wireless Sensors," in IEEE Transactions on Power Electronics, vol. 34, no. 10, pp. 9794-9805, Oct. 2019, doi: 10.1109/TPEL.2019.2894465.

[10] I. Khan, F. Belqasmi, R. Glitho, N. Crespi, M. Morrow and P. Polakos, "Wireless sensor network virtualization: A survey," in IEEE Communications Surveys \& Tutorials, vol. 18, no. 1, pp. 553-576, Firstquarter 2016, doi: 10.1109/COMST.2015.2412971.

[11] P. Park, S. Coleri Ergen, C. Fischione, C. Lu and K. H. Johansson, "Wireless Network Design for Control Systems: A Survey," in IEEE Communications Surveys \& Tutorials, vol. 20, no. 2, pp. 978-1013, Secondquarter 2018, doi: 10.1109/COMST.2017.2780114.

[12] S. H. R. Bukhari, M. H. Rehmani and S. Siraj, "A Survey of Channel Bonding for Wireless Networks and Guidelines of Channel Bonding for Futuristic Cognitive Radio Sensor Networks," in IEEE Communications Surveys \& Tutorials, vol. 18, no. 2, pp. 924-948, Secondquarter 2016, doi: 10.1109/COMST.2015.2504408.

[13] S. Movassaghi, M. Abolhasan, J. Lipman, D. Smith and A. Jamalipour, "Wireless Body Area Networks: A Survey," in IEEE Communications Surveys \& Tutorials, vol. 16, no. 3, pp. 1658-1686, Third Quarter 2014, doi: 10.1109/SURV.2013.121313.00064.

[14] S. Jayaprakasam, S. K. A. Rahim and C. Y. Leow, "Distributed and Collaborative Beamforming in Wireless Sensor Networks: Classifications, Trends, and Research Directions," in IEEE Communications Surveys \& Tutorials, vol. 19, no. 4, pp. 2092-2116, Fourthquarter 2017, doi: 10.1109/COMST.2017.2720690.

[15] S. Ezdiani, I. S. Acharyya, S. Sivakumar and A. Al-Anbuky, "Wireless Sensor Network Softwarization: Towards WSN Adaptive QoS," in IEEE Internet of Things Journal, vol. 4, no. 5, pp. 1517-1527, Oct. 2017, doi: 10.1109/JIOT.2017.2740423.

[16] Z. Ma, M. Xiao, Y. Xiao, Z. Pang, H. V. Poor and B. Vucetic, "HighReliability and Low-Latency Wireless Communication for Internet of Things: Challenges, Fundamentals, and Enabling Technologies," in IEEE Internet of Things Journal, vol. 6, no. 5, pp. 7946-7970, Oct. 2019, doi: 10.1109/JIOT.2019.2907245. 
[17] F. Engmann, F. A. Katsriku, J-D Abdulai, K. S. Adu-Manu, and F. K. Banaseka, "Prolonging the Lifetime of Wireless Sensor Networks: A Review of Current Techniques”, Hindawi, Wireless Communications and Mobile Computing, 2018.

[18] X. Liu, "Atypical Hierarchical Routing Protocols for Wireless Sensor Networks: A Review," in IEEE Sensors Journal, vol. 15, no. 10, pp. 5372-5383, Oct. 2015, doi: 10.1109/JSEN.2015.2445796.

[19] D. N. Sandeep and V. Kumar, "Review on Clustering, Coverage and Connectivity in Underwater Wireless Sensor Networks: A Communication Techniques Perspective," in IEEE Access, vol. 5, pp. 11176-11199, 2017, doi: 10.1109/ACCESS.2017.2713640.

[20] R. Asorey-Cacheda, A.-J. Garcia-Sanchez, F. Garcia-Sanchez, J. GarciaHaro, "A survey on non-linear optimization problems in wireless sensor networks”, Elsevier- Journal of Network and Computer Applications 82 (2017) 1-20.

[21] Z. Fei, B. Li, S. Yang, C. Xing, H. Chen and L. Hanzo, "A Survey of Multi-Objective Optimization in Wireless Sensor Networks: Metrics, Algorithms, and Open Problems," in IEEE Communications Surveys \& Tutorials, vol. 19, no. 1, pp. 550-586, Firstquarter 2017, doi: 10.1109/COMST.2016.2610578.

[22] L. Cao, Y. Cai and Y. Yue, "Swarm Intelligence-Based Performance Optimization for Mobile Wireless Sensor Networks: Survey, Challenges, and Future Directions," in IEEE Access, vol. 7, pp. 161524161553, 2019, doi: 10.1109/ACCESS.2019.2951370.

[23] R. A. Diab, N. Bastaki and A. Abdrabou, "A Survey on Routing Protocols for Delay and Energy-Constrained Cognitive Radio Networks," in IEEE Access, vol. 8, pp. 198779-198800, 2020, doi: 10.1109/ACCESS.2020.3035325.

[24] N.N. Srinidhi, S.M. Dilip Kumar, K.R. Venugopal, "Network optimizations in the Internet of Things: A review", ElsevierEngineering Science and Technology, an International Journal 22 (2019) $1-21$.

[25] S. Khisa and S. Moh, "Survey on Recent Advancements in EnergyEfficient Routing Protocols for Underwater Wireless Sensor Networks," in IEEE Access, vol. 9, pp. 55045-55062, 2021, doi: 10.1109/ACCESS.2021.3071490.

[26] A. J. Williams, M. F. Torquato, I. M. Cameron, A. A. Fahmy and J. Sienz, "Survey of Energy Harvesting Technologies for Wireless Sensor Networks," in IEEE Access, vol. 9, pp. 77493-77510, 2021, doi: 10.1109/ACCESS.2021.3083697.

[27] J. He et al., "Application of Game Theory in Integrated Energy System Systems: A Review," in IEEE Access, vol. 8, pp. 93380-93397, 2020, doi: 10.1109/ACCESS.2020.2994133.

[28] M. A. Abd, S. F. M. Al-Rubeaai, B. K. Singh, K. E. Tepe and R. Benlamri, "Extending Wireless Sensor Network Lifetime With Global Energy Balance," in IEEE Sensors Journal, vol. 15, no. 9, pp. 50535063, Sept. 2015, doi: 10.1109/JSEN.2015.2432114.

[29] S. Chowdhury, A. Benslimane and C. Giri, "Noncooperative Gaming for Energy-Efficient Congestion Control in 6LoWPAN," in IEEE Internet of Things Journal, vol. 7, no. 6, pp. 4777-4788, June 2020, doi: 10.1109/JIOT.2020.2969272.

[30] D. Lin and Q. Wang, "An Energy-Efficient Clustering Algorithm Combined Game Theory and Dual-Cluster-Head Mechanism for WSNs," in IEEE Access, vol. 7, pp. 49894-49905, 2019, doi: 10.1109/ACCESS.2019.2911190.

[31] L. Peizhe, W. Muqing, L. Wenxing and Z. Min, "A Game-Theoretic and Energy-Efficient Algorithm in an Improved Software-Defined Wireless Sensor Network," in IEEE Access, vol. 5, pp. 13430-13445, 2017, doi: 10.1109/ACCESS.2017.2727139.

[32] B. Shahrokhzadeh and M. Dehghan, "A Distributed Game-Theoretic Approach for Target Coverage in Visual Sensor Networks," in IEEE Sensors Journal, vol. 17, no. 22, pp. 7542-7552, 15 Nov.15, 2017, doi: 10.1109/JSEN.2017.2739183.

[33] M. Zayene, O. Habachi, V. Meghdadi, T. Ezzedine and J. P. Cances, "A Coalitional Game-Theoretic Framework for Cooperative Data Exchange Using Instantly Decodable Network Coding," in IEEE Access, vol. 7, pp. 26752-26765, 2019, doi: 10.1109/ACCESS.2019.2900982.

[34] S. Jeong, M. M. Tentzeris and S. Kim, "Machine Learning Approach for Wirelessly Powered RFID-Based Backscattering Sensor System," in
IEEE Journal of Radio Frequency Identification, vol. 4, no. 3, pp. 186194, Sept. 2020, doi: 10.1109/JRFID.2020.3004035.

[35] W. Li et al., "Vibrational Triboelectric Nanogenerator-Based Multinode Self-Powered Sensor Network for Machine Fault Detection," in IEEE/ASME Transactions on Mechatronics, vol. 25, no. 5, pp. 21882196, Oct. 2020, doi: 10.1109/TMECH.2020.2993336.

[36] Suxia Chen, Quanzheng Huang, Yang Zhang and Xin Li, "Double sink energy hole avoidance strategy for wireless sensor network", Eurasip Journal on Wireless Communication and Networking, 2020.

[37] Shu-Chuan Chu, Thi-Kien Dao, Jeng-Shyang Pan, and Trong-The Nguyen, "Identifying correctness data scheme for aggregating data in cluster heads of wireless sensor network based on naïve Bayes classification”, Eurasip Journal on Wireless Communication and Networking, 2020.

[38] Abdulaziz Y. Barnawi and Ismail M. Keshta, "Energy Management in Wireless Sensor Networks Based on Naive Bayes, MLP, and SVM Classifications: A Comparative Study”, Hindawi Publishing Corporation, Journal of Sensors, 2016.

[39] T. Fu, W. Peng and W. Lee, "Parallelizing Itinerary-Based KNN Query Processing in Wireless Sensor Networks," in IEEE Transactions on Knowledge and Data Engineering, vol. 22, no. 5, pp. 711-729, May 2010, doi: 10.1109/TKDE.2009.146.

[40] N. Marchang and R. Tripathi, "KNN-ST: Exploiting Spatio-Temporal Correlation for Missing Data Inference in Environmental Crowd Sensing," in IEEE Sensors Journal, vol. 21, no. 3, pp. 3429-3436, 1 Feb.1, 2021, doi: 10.1109/JSEN.2020.3024976.

[41] Y. Yilmaz, G. V. Moustakides and X. Wang, "Sequential and decentralized estimation of linear-regression parameters in wireless sensor networks," in IEEE Transactions on Aerospace and Electronic Systems, vol. 52, no. 1, pp. 288-306, February 2016, doi: 10.1109/TAES.2015.140665.

[42] D. Upadhyay, A. K. Dubey and P. S. Thilagam, "Application of NonLinear Gaussian Regression-Based Adaptive Clock Synchronization Technique for Wireless Sensor Network in Agriculture," in IEEE Sensors Journal, vol. 18, no. 10, pp. 4328-4335, 15 May15, 2018, doi: 10.1109/JSEN.2018.2818302.

[43] S. Shamshirband, D. Petković, H. Javidnia and A. Gani, "Sensor Data Fusion by Support Vector Regression Methodology-A Comparative Study," in IEEE Sensors Journal, vol. 15, no. 2, pp. 850-854, Feb. 2015, doi: 10.1109/JSEN.2014.2356501.

[44] Ni Guo, Weifeng Gui, Wei Chen, Xin Tian, Weiguo Qiu, Zijian Tian and Xiangyang Zhang, "Using improved support vector regression to predict the transmitted energy consumption data by distributed wireless sensor network" Eurasip Journal on Wireless Communication and Networking, 2020.

[45] B. Alotaibi, "Transportation Mode Detection by Embedded Sensors Based on Ensemble Learning," in IEEE Access, vol. 8, pp. 145552145563, 2020, doi: 10.1109/ACCESS.2020.3014901.

[46] D. R. Wijaya and F. Afianti, "Information-Theoretic Ensemble Feature Selection With Multi-Stage Aggregation for Sensor Array Optimization," in IEEE Sensors Journal, vol. 21, no. 1, pp. 476-489, 1 Jan.1, 2021, doi: 10.1109/JSEN.2020.3000756.

[47] R. R. Rout, M. S. Krishna and S. Gupta, "Markov Decision ProcessBased Switching Algorithm for Sustainable Rechargeable Wireless Sensor Networks," in IEEE Sensors Journal, vol. 16, no. 8, pp. 27882797, April15, 2016, doi: 10.1109/JSEN.2016.2519399.

[48] J. Zheng, S. Yang, X. Wang, X. Xia, Y. Xiao and T. Li, "A Decision Tree Based Road Recognition Approach Using Roadside Fixed 3D LiDAR Sensors," in IEEE Access, vol. 7, pp. 53878-53890, 2019, doi: 10.1109/ACCESS.2019.2912581.

[49] C. Lin, Y. C. Yang, J. Wang and Y. Yang, "A Wearable Sensor Module With a Neural-Network-Based Activity Classification Algorithm for Daily Energy Expenditure Estimation," in IEEE Transactions on Information Technology in Biomedicine, vol. 16, no. 5, pp. 991-998, Sept. 2012, doi: 10.1109/TITB.2012.2206602.

[50] K. Wu, F. Li, C. Tellambura and H. Jiang, "Optimal Selective Transmission Policy for Energy-Harvesting Wireless Sensors via Monotone Neural Networks," in IEEE Internet of Things Journal, vol. 6, no. 6, pp. 9963-9978, Dec. 2019, doi: 10.1109/JIOT.2019.2933579. 
[51] A. Mukherjee, D. K. Jain, P. Goswami, Q. Xin, L. Yang and J. J. P. C. Rodrigues, "Back Propagation Neural Network Based Cluster Head Identification in MIMO Sensor Networks for Intelligent Transportation Systems," in IEEE Access, vol. 8, pp. 28524-28532, 2020, doi: 10.1109/ACCESS.2020.2971969.

[52] L. Cheng, J. Hang, Y. Wang and Y. Bi, "A Fuzzy C-Means and Hierarchical Voting Based RSSI Quantify Localization Method for Wireless Sensor Network," in IEEE Access, vol. 7, pp. 47411-47422, 2019, doi: 10.1109/ACCESS.2019.2909974.

[53] W. Fei, B. Hexiang, L. Deyu and W. Jianjun, "Energy-Efficient Clustering Algorithm in Underwater Sensor Networks Based on Fuzzy C Means and Moth-Flame Optimization Method," in IEEE Access, vol. 8, pp. 97474-97484, 2020, doi: 10.1109/ACCESS.2020.2997066.

[54] M. Shi and L. Feng, "Plug-and-Play Prior Based on Gaussian Mixture Model Learning for Image Restoration in Sensor Network," in IEEE Access, vol. 6, pp. 78113-78122, 2018, doi: 10.1109/ACCESS.2018.2884795.

[55] R. Eguchi, B. Michael, M. Howard and M. Takahashi, "Shift-Adaptive Estimation of Joint Angle Using Instrumented Brace With Two Stretch Sensors Based on Gaussian Mixture Models," in IEEE Robotics and Automation Letters, vol. 5, no. 4, pp. 5881-5888, Oct. 2020, doi: 10.1109/LRA.2020.3010486.

[56] P. Liu, S. Nguang and A. Partridge, "Occupancy Inference Using Pyroelectric Infrared Sensors Through Hidden Markov Models," in IEEE Sensors Journal, vol. 16, no. 4, pp. 1062-1068, Feb.15, 2016, doi: 10.1109/JSEN.2015.2496154.

[57] M. Xu and Y. Wang, "Quantifying PM2.5 Concentrations From MultiWeather Sensors Using Hidden Markov Models," in IEEE Sensors Journal, vol. 16, no. 1, pp. 22-23, Jan.1, 2016, doi: 10.1109/JSEN.2015.2485665.

[58] T. Kim, L. F. Vecchietti, K. Choi, S. Lee and D. Har, "Machine Learning for Advanced Wireless Sensor Networks: A Review," in IEEE Sensors Journal, vol. 21, no. 11, pp. 12379-12397, 1 June1, 2021, doi: 10.1109/JSEN.2020.3035846.

[59] A. Ashiquzzaman, H. Lee, T. Um and J. Kim, "Energy-Efficient IoT Sensor Calibration With Deep Reinforcement Learning," in IEEE Access, vol. 8, pp. 97045-97055, 2020, doi: 10.1109/ACCESS.2020.2992853.

[60] H. Ke, J. Wang, H. Wang and Y. Ge, "Joint Optimization of Data Offloading and Resource Allocation With Renewable Energy Aware for IoT Devices: A Deep Reinforcement Learning Approach," in IEEE Access, vol. 7, pp. 179349-179363, 2019, doi: 10.1109/ACCESS.2019.2959348.

[61] K. K. Nguyen, T. Q. Duong, N. A. Vien, N. -A. Le-Khac and M. -N. Nguyen, "Non-Cooperative Energy Efficient Power Allocation Game in D2D Communication: A Multi-Agent Deep Reinforcement Learning Approach," in IEEE Access, vol. 7, pp. 100480-100490, 2019, doi: 10.1109/ACCESS.2019.2930115.

[62] B. O. Ayinde and A. Y. Barnawi, "Energy Conservation in Wireless Sensor Networks Using Partly-Informed Sparse Autoencoder," in IEEE Access, vol. 7, pp. 63346-63360, 2019, doi: 10.1109/ACCESS.2019.2917322.

[63] N. Dinh, T. Gu and Y. Kim, "Rendezvous Cost-Aware Opportunistic Routing in Heterogeneous Duty-Cycled Wireless Sensor Networks," in IEEE Access, vol. 7, pp. 121825-121840, 2019, doi: 10.1109/ACCESS.2019.2937252.

[64] J. Kang, J. Kim, M. Kim and M. Sohn, "Machine Learning-Based Energy-Saving Framework for Environmental States-Adaptive Wireless Sensor Network," in IEEE Access, vol. 8, pp. 69359-69367, 2020, doi: 10.1109/ACCESS.2020.2986507.

[65] J. C. Kwan, J. M. Chaulk and A. O. Fapojuwo, "A Coordinated Ambient/Dedicated Radio Frequency Energy Harvesting Scheme Using Machine Learning," in IEEE Sensors Journal, vol. 20, no. 22, pp. 1380813823, 15 Nov.15, 2020, doi: 10.1109/JSEN.2020.3003931.

[66] R. Du, M. Xiao and C. Fischione, "Optimal Node Deployment and Energy Provision for Wirelessly Powered Sensor Networks," in IEEE Journal on Selected Areas in Communications, vol. 37, no. 2, pp. 407423, Feb. 2019, doi: 10.1109/JSAC.2018.2872380.
[67] W. Feng et al., "Joint Energy-Saving Scheduling and Secure Routing for Critical Event Reporting in Wireless Sensor Networks," in IEEE Access, vol. 8, pp. 53281-53292, 2020, doi: 10.1109/ACCESS.2020.2981115.

[68] L. Huang, J. Wang, E. Kung, Y. Mo, J. Wu and L. Shi, "Stochastic Event-Based Sensor Schedules for Remote State Estimation in Cognitive Radio Sensor Networks," in IEEE Transactions on Automatic Control, vol. 66, no. 5, pp. 2407-2414, May 2021, doi: 10.1109/TAC.2020.3007510.

[69] Z. Li, J. Xie, H. Zhang, H. Xiang and Z. Zhang, "Adaptive Sensor Scheduling and Resource Allocation in Netted Collocated MIMO Radar System for Multi-Target Tracking," in IEEE Access, vol. 8, pp. 109976109988, 2020, doi: 10.1109/ACCESS.2020.3001358.

[70] X. Liu, Y. Gao and F. Hu, "Optimal Time Scheduling Scheme for Wireless Powered Ambient Backscatter Communications in IoT Networks," in IEEE Internet of Things Journal, vol. 6, no. 2, pp. 22642272, April 2019, doi: 10.1109/JIOT.2018.2889700.

[71] H. Shi, M. Zheng, W. Liang and J. Zhang, "Convergecast Scheduling for Industrial Wireless Sensor Networks With Local Available Channel Sets," in IEEE Sensors Journal, vol. 19, no. 22, pp. 10764-10772, 15 Nov.15, 2019, doi: 10.1109/JSEN.2019.2929672.

[72] Runze Wan, Naixue Xiong and Nguyen The Loc, "An energy-efficient sleep scheduling mechanism with similarity measure for wireless sensor networks”, Human-Centric Computing and Information Sciences, 2018

[73] H. Wang, S. Tan, Y. Zhu and M. Li, "Deterministic Scheduling With Optimization of Average Transmission Delays in Industrial Wireless Sensor Networks," in IEEE Access, vol. 8, pp. 18852-18862, 2020, doi: 10.1109/ACCESS.2020.2968491.

[74] W. Yang, Y. Zhang, C. Yang, Z. Zuo and X. Wang, "Online Power Scheduling for Distributed Filtering Over an Energy-Limited Sensor Network," in IEEE Transactions on Industrial Electronics, vol. 65, no. 5, pp. 4216-4226, May 2018, doi: 10.1109/TIE.2017.2756594.

[75] H. Zhang, J. Xie, J. Shi, Z. Zhang and X. Fu, "Sensor Scheduling and Resource Allocation in Distributed MIMO Radar for Joint Target Tracking and Detection," in IEEE Access, vol. 7, pp. 62387-62400, 2019, doi: 10.1109/ACCESS.2019.2916334.

[76] F. Ait Aoudia, M. Gautier and O. Berder, "RLMan: An Energy Manager Based on Reinforcement Learning for Energy Harvesting Wireless Sensor Networks," in IEEE Transactions on Green Communications and Networking, vol. 2, no. 2, pp. 408-417, June 2018, doi: 10.1109/TGCN.2018.2801725.

[77] Z. J. Chew, T. Ruan and M. Zhu, "Energy Savvy Network Joining Strategies for Energy Harvesting Powered TSCH Nodes," in IEEE Transactions on Industrial Informatics, vol. 17, no. 2, pp. 1505-1514, Feb. 2021, doi: 10.1109/TII.2020.3005196.

[78] J. Hu, J. Luo, Y. Zheng and K. Li, "Graphene-Grid Deployment in Energy Harvesting Cooperative Wireless Sensor Networks for Green IoT," in IEEE Transactions on Industrial Informatics, vol. 15, no. 3, pp. 1820-1829, March 2019, doi: 10.1109/TII.2018.2871183.

[79] M. Koca, G. Gurbilek, B. Soner and S. Coleri, "Empirical Feasibility Analysis for Energy Harvesting Intravehicular Wireless Sensor Networks," in IEEE Internet of Things Journal, vol. 8, no. 1, pp. 179186, 1 Jan.1, 2021, doi: 10.1109/JIOT.2020.3001992.

[80] T. Ruan, Z. J. Chew and M. Zhu, "Energy-Aware Approaches for Energy Harvesting Powered Wireless Sensor Nodes," in IEEE Sensors Journal, vol. 17, no. 7, pp. 2165-2173, 1 April1, 2017, doi: 10.1109/JSEN.2017.2665680.

[81] C. Wang, J. Li, Y. Yang and F. Ye, "Combining Solar Energy Harvesting with Wireless Charging for Hybrid Wireless Sensor Networks," in IEEE Transactions on Mobile Computing, vol. 17, no. 3, pp. 560-576, 1 March 2018, doi: 10.1109/TMC.2017.2732979.

[82] Y. Xiong, G. Chen, M. Lu, X. Wan, M. Wu and J. She, "A Two-Phase Lifetime-Enhancing Method for Hybrid Energy-Harvesting Wireless Sensor Network," in IEEE Sensors Journal, vol. 20, no. 4, pp. 19341946, 15 Feb.15, 2020, doi: 10.1109/JSEN.2019.2948620.

[83] Y. -H. Zhu, S. Gong, K. Chi, Y. Li and Y. Fang, "Optimizing Superframe and Data Buffer to Achieve Maximum Throughput for 802.15.4-Based Energy Harvesting Wireless Sensor Networks," in IEEE Internet of Things Journal, vol. 8, no. 5, pp. 3689-3704, 1 March1, 2021, doi: 10.1109/JIOT.2020.3024615. 
[84] U. F. Abbasi, N. Haider, A. Awang and K. S. Khan, "Cross-Layer MAC/Routing Protocol for Reliable Communication in Internet of Health Things," in IEEE Open Journal of the Communications Society, vol. 2, pp. 199-216, 2021, doi: 10.1109/OJCOMS.2020.3047888.

[85] M. Z. Hasan, F. Al-Turjman and H. Al-Rizzo, "Analysis of Cross-Layer Design of Quality-of-Service Forward Geographic Wireless Sensor Network Routing Strategies in Green Internet of Things," in IEEE Access, vol. 6, pp. 20371-20389, 2018, doi: 10.1109/ACCESS.2018.2822551.

[86] M. J. Herrmann and G. G. Messier, "Cross-Layer Lifetime Optimization for Practical Industrial Wireless Networks: A Petroleum Refinery Case Study," in IEEE Transactions on Industrial Informatics, vol. 14, no. 8, pp. 3559-3566, Aug. 2018, doi: 10.1109/TII.2018.2819678.

[87] M. V. Ngo, Q. D. La, D. Leong, T. Q. S. Quek and H. Shin, "User Behavior Driven MAC Scheduling for Body Sensor Networks: A CrossLayer Approach," in IEEE Sensors Journal, vol. 19, no. 17, pp. 77557765, 1 Sept.1, 2019, doi: 10.1109/JSEN.2019.2915635.

[88] S. M. Shimly, D. B. Smith and S. Movassaghi, "Experimental Analysis of Cross-Layer Optimization for Distributed Wireless Body-to-Body Networks," in IEEE Sensors Journal, vol. 19, no. 24, pp. 12494-12509, 15 Dec.15, 2019, doi: 10.1109/JSEN.2019.2937356.

[89] Z. Sun et al., "An Energy-Efficient Cross-Layer-Sensing Clustering Method Based on Intelligent Fog Computing in WSNs," in IEEE Access, vol. 7, pp. 144165-144177, 2019, doi: 10.1109/ACCESS.2019.2944858.

[90] W. Zhang, X. Wei, G. Han and X. Tan, "An Energy-Efficient Ring Cross-Layer Optimization Algorithm for Wireless Sensor Networks," in IEEE Access, vol. 6, pp. 16588-16598, 2018, doi: 10.1109/ACCESS.2018.2809663.

[91] M. Y. Arafat and S. Moh, "Bio-Inspired Approaches for EnergyEfficient Localization and Clustering in UAV Networks for Monitoring Wildfires in Remote Areas," in IEEE Access, vol. 9, pp. 18649-18669, 2021, doi: 10.1109/ACCESS.2021.3053605.

[92] L. Cao, Y. Cai, Y. Yue, S. Cai and B. Hang, "A Novel Data Fusion Strategy Based on Extreme Learning Machine Optimized by Bat
Algorithm for Mobile Heterogeneous Wireless Sensor Networks," in IEEE Access, vol. 8, pp. 16057-16072, 2020, doi: 10.1109/ACCESS.2020.2967118.

[93] F. Dai, M. Chen, X. Wei and H. Wang, "Swarm Intelligence-Inspired Autonomous Flocking Control in UAV Networks," in IEEE Access, vol. 7, pp. 61786-61796, 2019, doi: 10.1109/ACCESS.2019.2916004.

[94] M. Faheem et al., "FFRP: Dynamic Firefly Mating Optimization Inspired Energy Efficient Routing Protocol for Internet of Underwater Wireless Sensor Networks," in IEEE Access, vol. 8, pp. 39587-39604, 2020, doi: 10.1109/ACCESS.2020.2976105.

[95] Y. Feng, S. Zhao and H. Liu, "Analysis of Network Coverage Optimization Based on Feedback K-Means Clustering and Artificial Fish Swarm Algorithm," in IEEE Access, vol. 8, pp. 42864-42876, 2020, doi: 10.1109/ACCESS.2020.2970208.

[96] M. Z. Hasan and H. Al-Rizzo, "Optimization of Sensor Deployment for Industrial Internet of Things Using a Multiswarm Algorithm," in IEEE Internet of Things Journal, vol. 6, no. 6, pp. 10344-10362, Dec. 2019, doi: 10.1109/JIOT.2019.2938486.

[97] E. H. Houssein, M. R. Saad, K. Hussain, W. Zhu, H. Shaban and M. Hassaballah, "Optimal Sink Node Placement in Large Scale Wireless Sensor Networks Based on Harris' Hawk Optimization Algorithm," in IEEE Access, vol. 8, pp. 19381-19397, 2020, doi: 10.1109/ACCESS.2020.2968981.

[98] A. Mukherjee, P. Goswami, Z. Yan, L. Yang and J. J. P. C. Rodrigues, "ADAI and Adaptive PSO-Based Resource Allocation for Wireless Sensor Networks," in IEEE Access, vol. 7, pp. 131163-131171, 2019, doi: 10.1109/ACCESS.2019.2940821.

[99] W. Osamy, A. A. El-Sawy and A. Salim, "CSOCA: Chicken Swarm Optimization Based Clustering Algorithm for Wireless Sensor Networks," in IEEE Access, vol. 8, pp. 60676-60688, 2020, doi: 10.1109/ACCESS.2020.2983483.

[100]Z. Wei, M. Li, Z. Wei, L. Cheng, Z. Lyu and F. Liu, "A Novel OnDemand Charging Strategy Based on Swarm Reinforcement Learning in WRSNs," in IEEE Access, vol. 8, pp. 84258-84271, 2020, doi: 10.1109/ACCESS.2020.2992127. 